\title{
Surgical Management of Pulmonary Mucormycosis in Third-Trimester Pregnancy
}

\author{
Joel L. Ramirez ${ }^{1} \quad$ Anatoly Urisman ${ }^{2} \quad$ Jasleen Kukreja ${ }^{1} \quad$ Johannes R. Kratz ${ }^{1}$ \\ ${ }^{1}$ Department of Surgery, University of California, San Francisco, \\ San Francisco, California, United States \\ 2 Department of Pathology, University of California, San Francisco, \\ San Francisco, California, United States \\ Address for correspondence Johannes R. Kratz, MD, Department of \\ Surgery, University of California, 500 Parnassus Ave MUW-405, San \\ Francisco, CA 94143, United States \\ (e-mail: Johannes.kratz@ucsf.edu).
}

Thorac Cardiovasc Surg Rep 2018;7:e27-e29.

\begin{abstract}
Keywords

- pregnancy

- mucormycosis

- pulmonary mucromycosis

Background Pulmonary mucormycosis is a rare fungal infection that carries a high mortality. Given the rarity of this disease, its management has not been well established.

Case Description We report a 36-year-old female presenting with right middle and lower lobe pulmonary mucormycosis during the third trimester of pregnancy. Diagnosis was established using chest computed tomography followed by bronchoalveolar lavage and lung biopsy. Prompt initiation of amphotericin B and right middle and lower lobe lobectomy resulted in maternal survival and fetal viability.

Conclusion This favorable outcome is attributed to extensive communication between treatment teams in addition to comprehensive surgical planning.
\end{abstract}

\section{Introduction}

Mucormycosis is a rare fungal infection caused by fungi in the order of Mucorales, and is known to cause multiple locally invasive and often devastating syndromes in humans. Given the rarity of pulmonary mucormycosis, its incidence is not welldocumented, and incidence reports vary widely. ${ }^{1}$ We report the presentation, diagnosis, and surgical management of pulmonary mucormycosis during the third trimester of pregnancy.

\section{Case Description}

A 36-year-old gravida 4, para 6 female with a history of poorly controlled insulin-dependent type 2 diabetes initially presented at 28 weeks of gestation to a community hospital with complaints of weakness, shortness of breath, and hemoptysis. One month prior, she was admitted to a community hospital in diabetic ketoacidosis, during which she was persistently febrile and underwent a computed tomography (CT) scan of her chest that demonstrated a right lower lobe (RLL) infiltrate. A respiratory culture subsequently grew methicillin-resistant staphylococcus aureus, and she was treated with vancomycin and discharged home. However, she continued to feel poorly, which prompted her readmission to the same hospital. During her second admission, a chest CT demonstrated progression of the RLL infiltrate and presence of a new pleural effusion. She underwent a bronchoscopy with biopsy and bronchoalveolar lavage (BAL) that confirmed the diagnosis of pulmonary mucormycosis. She was subsequently transferred to our institution for further evaluation and management.

Chest CT demonstrated extensive necrosis and a $6.1 \mathrm{~cm}$ abscess in the RLL, as well as infiltrates in her right middle and left lower lobe (-Figs. 1 and 2). She was immediately initiated on intravenous liposomal amphotericin B. After consultation with multiple specialty services including obstetrics, maternal and fetal medicine, critical care, and infectious disease, the thoracic surgery team recommended surgical intervention in the presence of the obstetric and fetal medicine teams in case intraoperative cesarian section was necessary for maternal safety.

On hospital day 5, and at 29 weeks of gestation, she was taken to the operating room. Prior to the start of the procedure, a preoperative meeting detailing the operative plan and potential emergency scenarios was conducted in the presence received

March 20, 2018 accepted after revision May 2, 2018
DOI https://doi.org/

10.1055/s-0038-1660806. ISSN 2194-7635.
๑) 2018 Georg Thieme Verlag KG
Stuttgart · New York

License terms

(®) $\Theta \circledast$ 


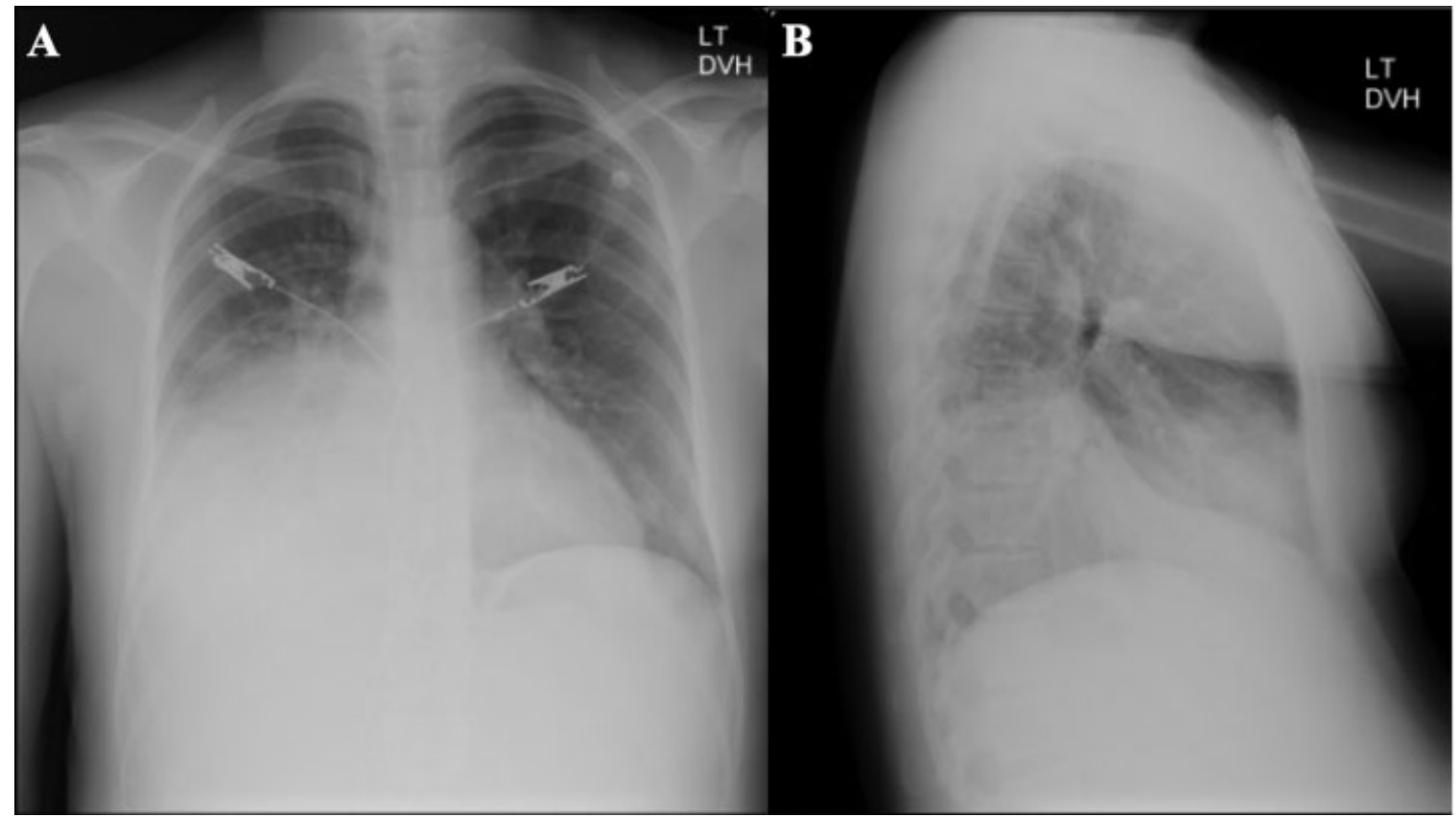

Fig. 1 Admission (A) anterior-posterior and (B) lateral chest X-ray demonstrating consolidation and effusion.

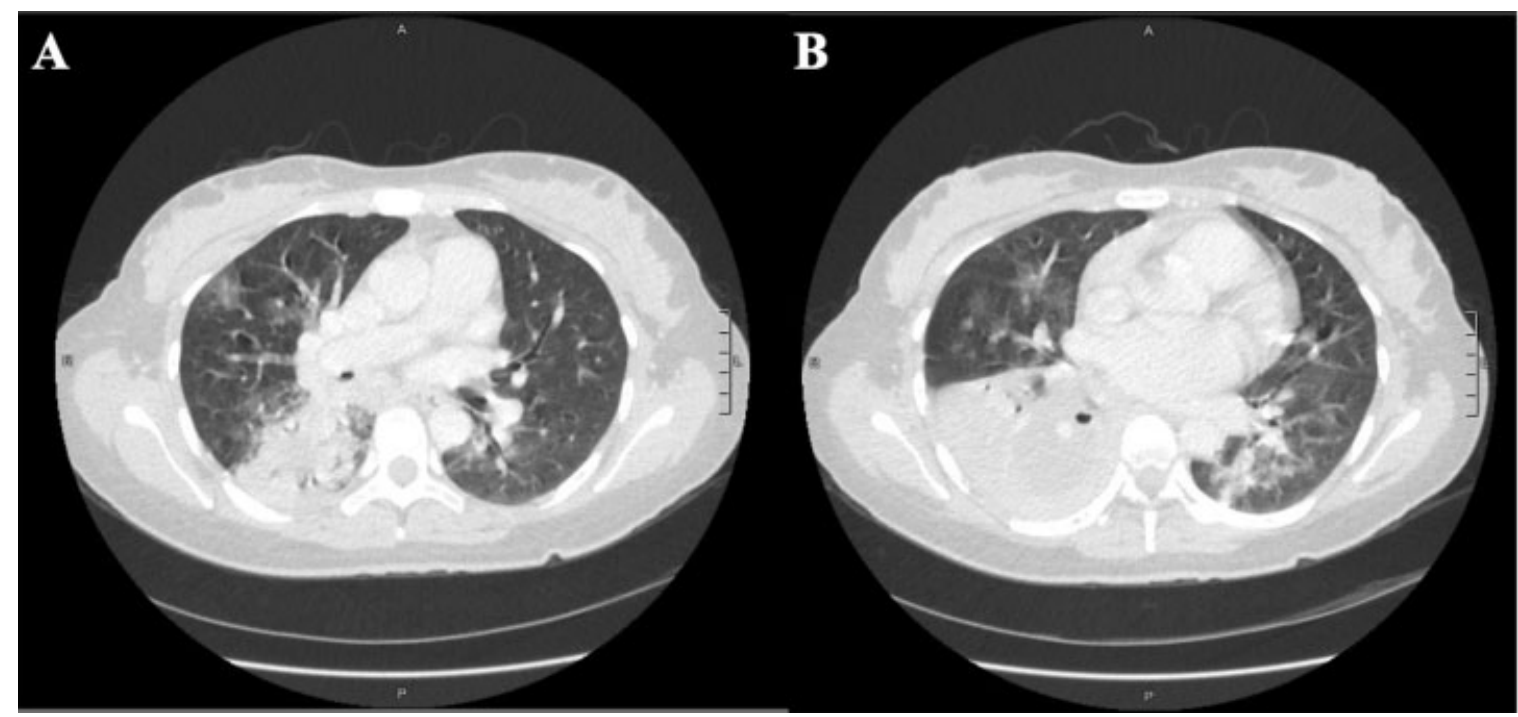

Fig. 2 Admission chest computed tomography scan demonstrating necrosis and abscess of the (A) right middle lobe and (B) right lower lobe.

of the surgical, obstetrics, pediatrics, and anesthesia teams. Flexible bronchoscopy demonstrated purulent secretions in the right airways and a white plaque, suspicious for fungal invasion, at the take-off of the middle lobe bronchus. The patient was placed in the left lateral decubitus position and the patient's hip was opened to allow access to the abdomen and pelvis in case of obstetric emergency. A standard posterolateral thoracotomy was performed, revealing a large fungal mass involving the right lower and middle lobes without signs of chest wall invasion. A right lower and middle bilobectomy was performed and an intercostal muscle flap was mobilized and fixed to the divided bronchus intermedius. A postoperative ultrasound demonstrated a viable fetus without any signs of distress.
Initially, the patient's postoperative course was complicated by a prolonged air leak and subcutaneous emphysema, requiring the placement of a second pleural pigtail catheter. Her air leak resolved on its own without further intervention and all chest tubes and catheters were removed by postoperative day 14 . She also developed a type 1 renal tubular acidosis secondary to amphotericin B and was switched to posaconazole, which she continued until delivery. On postoperative day 15 , she was transferred to the antepartum unit and subsequently discharged back to her home institution on postoperative day 24 . She delivered a healthy baby via cesarian section per patient preference at 37 weeks gestation without further complications from either her operation or pregnancy. 


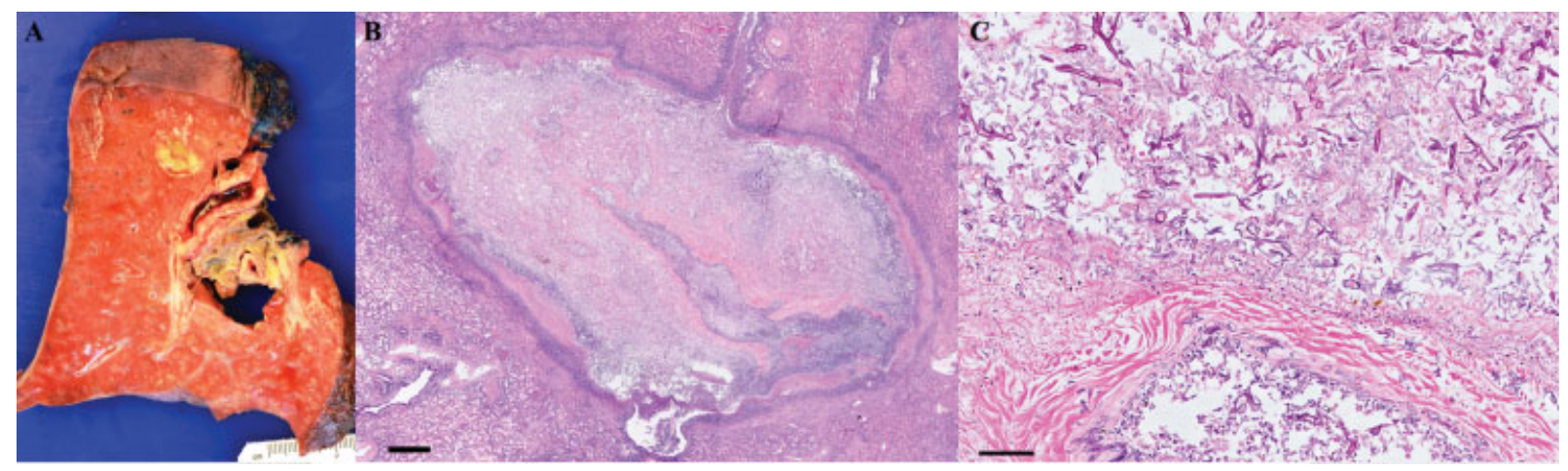

Fig. 3 Pathologic findings. (A) Right lower lobe gross findings. (B) Histologic findings, low magnification (hematoxylin and eosin; bar $=1 \mathrm{~mm}$ ). (C) Histologic findings, high magnification (hematoxylin and eosin; bar $=0.1 \mathrm{~mm}$ ).

On pathologic examination, the RLL specimen demonstrated a large perihilar airway-centered area of cavitation with surrounding fibrosis and distal airway impaction by thick mucus, as well as several rounded foci of apparent parenchymal necrosis with yellow mucoid appearance on cut surface (-Fig. 3A). The histologic sections corresponding to the areas of necrosis revealed consolidated parenchyma with large central liquefactive-type necrosis surrounded by organizing granulation tissue and fibrosis (-Fig. 3B). Numerous fungal hyphae were present in the areas of necrosis, with widespread tissue and angioinvasion (-Fig. 3C). The morphologic features of the fungal hyphae were consistent with zygomycetes (Mucorales or Rhizopus species).

\section{Discussion}

Lungs are the second most common site of mucormycosis infection. ${ }^{2}$ Risk factors for mucormycosis include neutropenia, chemotherapy, hematopoietic stem cell transplant, malignant hematologic disease, poorly controlled diabetes mellitus, prolonged steroid use, and an immunocompromised state., Pregnancy is not a recognized risk factor for pulmonary mucormycosis; however, the mild immunocompromised state of pregnancy ${ }^{4}$ may have increased this patient's overall risk for mucormycosis when combined with her history of poorly controlled diabetes. If left untreated, pulmonary mucormycosis progresses rapidly and has been reported to spread to the chest wall, mediastinum, heart, or hematogenously to other organs. ${ }^{3,5}$ Overall mortality for pulmonary mucormycosis is extremely high, particularly in immunocompromised patients. $^{3}$

Pulmonary mucormycosis commonly presents as a necrotizing pneumonia with symptoms including cough, fever, hemoptysis, and chest pain. ${ }^{3}$ These symptoms are all fairly nonspecific and are difficult to differentiate from other pulmonary pathologies. As in this case, patients typically do not respond to antibiotic treatment and may develop worsening radiographic features. Additionally, radiographic features of pulmonary mucormycosis are highly variable and nonspecific, including infiltration, consolidation, nodules, cavitations, hilar lymphadenopathy, halo sign, and pleural effusions. ${ }^{6}$ Diagnosis can be challenging; current guidelines recommend combining imaging techniques (high resolution chest $\mathrm{CT}$ ) with histopathologic findings (sputum, culture, BAL, biopsy).

Once diagnosed, pulmonary mucormycosis requires aggressive medical and surgical treatment in addition to addressing underlying predisposing factors. Early initiation of amphotericin B and surgical debridement are essential mainstays of treatment, and have been reported to result in cure of disease. ${ }^{3}$ In our case, although lobectomy during pregnancy carries both maternal and fetal risks, the risk associated with treating with medical therapy alone were unacceptably high. To reduce maternal and fetal risks, surgical coordination with obstetric and pediatric teams ensured that specialty-specific care was available to intervene intraoperatively if needed. The patient was also positioned to allow for quick and immediate access to the abdomen and pelvis if necessary. Betamethasone was not administered to expedite fetal lung maturation in case of immediate delivery due to concern that the corticosteroids would impair wound healing and increase the risk of recurrent mucormycosis postoperatively.

The successful outcome for both the mother and fetus in this case was likely due to rapid surgical intervention upon transfer to our institution, careful and methodical coordination of care between multiple interdisciplinary teams (thoracic surgery, obstetrics, fetal medicine, critical care, and infectious disease), and close attention to postoperative care in a monitored setting.

\section{References}

1 Petrikkos G, Skiada A, Lortholary O, Roilides E, Walsh TJ, Kontoyiannis DP. Epidemiology and clinical manifestations of mucormycosis. Clin Infect Dis 2012;54(Suppl 1):S23-S34

2 Torres-Narbona M, Guinea J, Martínez-Alarcón J, Muñoz P, Gadea I, Bouza E; MYCOMED Zygomycosis Study Group. Impact of zygomycosis on microbiology workload: a survey study in Spain. J Clin Microbiol 2007;45(06):2051-2053

3 Tedder M, Spratt JA, Anstadt MP, Hegde SS, Tedder SD, Lowe JE. Pulmonary mucormycosis: results of medical and surgical therapy. Ann Thorac Surg 1994;57(04):1044-1050

4 Munoz-Suano A, Hamilton AB, Betz AG. Gimme shelter: the immune system during pregnancy. Immunol Rev 2011;241(01):20-38

5 Connor BA, Anderson RJ, Smith JW. Mucor mediastinitis. Chest 1979;75(04):525-526

6 McAdams HP, Rosado de Christenson M, Strollo DC, Patz EF Jr. Pulmonary mucormycosis: radiologic findings in 32 cases. AJR Am J Roentgenol 1997;168(06):1541-1548 